\title{
Successful Treatment of Tinea Capitis Caused by Microsporum canis Using Griseofulvin
}

\author{
Brigita Ika Rosdiana ${ }^{\mathrm{a}}$, Yuri Widia ${ }^{\mathrm{b}}$, Linda Astari ${ }^{\mathrm{c}}$, Evy Ervianti ${ }^{\mathrm{d}}$, Dwi \\ Murtiastutik $^{\mathrm{e}}$
}

a. Department of Dermatology and Venereology, Faculty of Medicine, Universitas Airlangga - Dr. Soetomo General Hospital, Surabaya, Indonesia, dvikaros@gmail.com

b. Department of Dermatology and Venereology, Faculty of Medicine, Universitas Airlangga - Dr. Soetomo General Hospital, Surabaya, Indonesia, widia_yuri@yahoo.com

c. Department of Dermatology and Venereology, Faculty of Medicine, Universitas Airlangga - Dr. Soetomo General Hospital, Surabaya, Indonesia, lindaaastari@yahoo.com

d. Department of Dermatology and Venereology, Faculty of Medicine, Universitas Airlangga - Dr. Soetomo General Hospital, Surabaya, Indonesia, evy_if@yahoo.co.id

e. Department of Dermatology and Venereology, Faculty of Medicine, Universitas Airlangga - Dr. Soetomo General Hospital, Surabaya, Indonesia

Corresponding author:

Dr. Dwi Murtiastutik

Department of Dermatology and Venereology, Faculty of Medicine, Universitas

Airlangga - Dr. Soetomo General Hospital, Surabaya, Indonesia

Mayjend Prof. Dr. Moestopo Street No. 6-8, Airlangga, Gubeng, Surabaya, East Java, Indonesia 60285

E-mail: dwimurtiastutik@yahoo.co.id

\begin{abstract}
Tinea capitis is a scalp and hair follicle dermatophyte infection caused by Trichophyton $s p$. and Microsporum sp. that more commonly affect children than adults. Microsporum canis is the most common dermatophyte causing tinea capitis reported in Asia that respond well to griseofulvin. We report one case of an 8-year-old child with a history of patchy scaling lesion on the head, alopecia, and pruritus. Dermatological examination
\end{abstract}


revealed multiple scaly erythematous macules, circular shape, sharply marginated, with a diameter of 2-6 cm, along with alopecia in each lesion. $\mathrm{KOH}$ preparation, wood lamp examination, trichoscopy, and fungal culture were performed. The diagnosis of tinea capitis grey patch type with an etiology of Microsporum canis was established. The patient was significantly improved clinically and mycologically with four weeks course of oral griseofulvin and adjuvant treatment using ketoconazole $2 \%$ scalp solution. Griseofulvin is effective to treat tinea capitis caused by Microsporum sp. Comprehensive examination in tinea capitis is necessary to confirm the diagnosis and the etiology as well to determine the best treatment for the case.

\section{Keywords}

children; dermatophyte infection; griseofulvin; Microsporum; tinea capitis

\section{Introduction}

Tinea capitis is a dermatophyte infection that affects the scalp, hair follicles, and intervening skin. ${ }^{1}$ The incidence of tinea capitis globally is approximately $28 \%$. Although it can affect any age group, it remains a common dermatophyte infection affecting primarily children, among $4-14$ years old. ${ }^{2}$

Tinea capitis is caused by various dermatophytes classified into three genera: Trichophyton sp., Microsporum sp., and Epidermophyton. They may be anthropophilic (usually infects humans and rarely affects animals), zoophilic (infects animals but can be transmitted to humans), and geophilic (inhabit soil and infect both humans and animals) based on their host preference and natural habitat. ${ }^{3}$ The etiology of tinea capitis varies in different geographic regions, and this predominant causative agent is dependent on climates, socioeconomic condition, immune status of the host, hygiene, drug resistance, 
and dermatophyte characteristic factors. ${ }^{4}$ The leading etiological zoophilic agent worldwide reported is Microsporum canis, while Trichopython violaceoum and Trichopython tonsurans are the most frequent anthropophilic causative agents. ${ }^{3}$ Therapeutic regimen options for tinea capitis in children, including griseofulvin, terbinafine, itraconazole, and fluconazole. Griseofulvin has been reported to be more effective in treating tinea capitis caused by Microsporum $s p .^{5}$

\section{Case History}

An eight-year-old boy presented to the dermatology outpatient clinic of Dr. Soetomo General Hospital with chief complaint of scaling on the head, patchy alopecia, and pruritus. His first visit to the hospital was October 1st, 2019. At first, the patient felt an itchy sensation and localized hair loss on the scalp one month before the first visit. Two weeks before he came to the hospital, he went to a general practitioner and got steroid ointment and shampoo, but there was no improvement. One week before the first visit, white scales were noted on his scalp. He had never experienced this condition before, and no one in the same household was experiencing any skin or scalp problems. The mother reported that their neighbour has a cat as a pet and frequently interacts with the child. Physical examination showed a normal body mass index and stable vital sign. Dermatological examination revealed multiple scaly erythematous macules, circular shape, sharply marginated, with a diameter of 2-6 cm, along with alopecia in each lesion (Fig. 1a). There was no palpable lymph node enlargement in the retroauricular region. Supportive examination using $\mathrm{KOH}$ preparation was performed, with the result of ectothrix pattern in the hair shaft. Wood lamp examination showed positive yellow-green fluorescence. Trichoscopy of the lesions showed the pattern of diffuse scaling, 
perifollicular scaling, morse code-like hair, zigzag hair, black dot, broken hair, and comma hair (Fig. 2). Fungal culture results revealed the species of Microsporum canis. Based on history taking, clinical manifestation, and supportive examination, the diagnosis of tinea capitis grey patch type was established. The patient was treated using griseofulvin $250 \mathrm{mg}$ per day for four weeks with adjuvant treatment using ketoconazole $2 \%$ scalp solution. Patient's condition was significantly improved both clinically and mycologically in two months. There were no adverse effects to the child reported by the parents.

\section{Discussion}

Tinea capitis, also known as ringworm, is a dermatophyte infection of the hair and the scalp. ${ }^{1,3}$ The clinical manifestation can be classified as non-inflammatory and inflammatory type. The non-inflammatory type is often uncomplicated with the classic presentation that includes one or more scaly patches of alopecia with black dots (broken hair at the skin line) or grey patch. In contrast, the inflammatory type (kerion) usually takes the form of a painful pustule and plaques and is followed by scarring alopecia as a complication. ${ }^{1,6}$ The diagnosis of tinea capitis can be made with clinical suspicion, especially if a child presents with patchy alopecia that is followed by scaling, erythema, or pustule. However, sometimes determining the diagnosis is difficult in cases with mild symptoms. Clinical manifestations that resemble another differential diagnosis like alopecia areata, trichotillomania, or seborrheic dermatitis also make it harder to diagnose tinea capitis. ${ }^{6,7}$ Based on the signs and symptoms, this case was classified as noninflammatory type, with the specific grey patch type. Non-inflammatory tinea capitis is more common than the inflammatory type. The grey patch type is commonly seen with 
an anthropophilic organism such as Microsporum canis and Microsporum audouinii. These two organisms cause an ectothrix pattern that will fluoresce bright green colour in the Wood light examination. ${ }^{1,6}$ On physical examination, no lymphadenopathy was found. Lymphadenopathy generally presents in a child with tinea capitis. However, this should not delay the diagnosis of tinea capitis as lymphadenopathy can also occur in nonfungal disease. ${ }^{6}$ Moreover, if the infection signs are notably apparent, then the suspicion towards tinea capitis should be established. Trichoscopy was performed to ensure the suspicion of tinea capitis and to exclude the differential diagnosis. Trichoscopy is an easy, fast, and non-invasive diagnosis tool suitable for pediatric patients and can be useful for the diagnosis before being confirmed by the mycological culture result so the treatment would not be delayed by the diagnostic process, considering the long duration of therapy for fungal infections. ${ }^{8}$ The characteristic trichoscopy findings of Microsporum tinea capitis include morse code-like hairs, bent hair, zigzag hair, and diffuse scaling that were also present in this case. ${ }^{9}$

Some physicians diagnose tinea capitis based on clinical findings if the signs and symptoms are apparent and typical. However, additional laboratory tests such as $\mathrm{KOH}$ examination and mycological culture are still essential to determine the etiologic agent and treatment options (e.g. determine the effective antifungal type in certain dermatophytes; for example, griseofulvin is more effective in Microsporum than Trichophyton) ${ }^{6,10}$ In many tinea capitis cases, the clinical aspects might not be associated with the expected agent; thus, the fungal culture is still necessary as a gold standard diagnostic test. ${ }^{11}$ The clinical and supportive findings in this case then finally were being confirmed with the fungal culture result, which showed spindle-shaped macroconidia with terminal knobs and more than 6 cells leading to the species Microsporum canis (Fig 
3). Microsporum canis is zoophilic; thus, we might suspect the transmission came from the cat that frequently interacted with the patient as cats and dogs may play a role as reservoir or source of infection of Microsporum canis, and the transmission between human is considered rare. ${ }^{12}$ This finding is in line with a previous 5-year retrospective study by Sari et al. in Indonesia which showed that Microsporum canis was the most common causative species of tinea capitis that affect the age group of $0-14$ years old. ${ }^{13}$ Systemic antifungal drugs are required to treat tinea capitis because topical agents do not penetrate well to hair shafts and follicles. ${ }^{6,14}$ Topical treatment like topical selenium sulphide or ketoconazole may be used as an adjunct to systemic treatments. It has proven beneficial to reduce the viable spores responsible for the contagion and reinfection that may shorten the cure rate. ${ }^{15}$ Oral antifungal treatment, griseofulvin, is considered the most effective therapy for Microsporum tinea capitis due to griseofulvin's ability to concentrate in sweat since the location of the infection is ectothrix, in which the drug must reach the location of infection through sweat or sebum. ${ }^{5}$ Griseofulvin also has a good safety profile with lower adverse effects. The most common side effects are headache and gastrointestinal discomfort, that is usually mild and disappear along with the treatment. ${ }^{5}$

\section{Conclusion}

Griseofulvin is effective to treat tinea capitis caused by Microsporum $s p$. Comprehensive examination in tinea capitis is necessary to confirm the diagnosis and the etiology as well, in order to choose the best treatment for the case. 


\section{Figures}

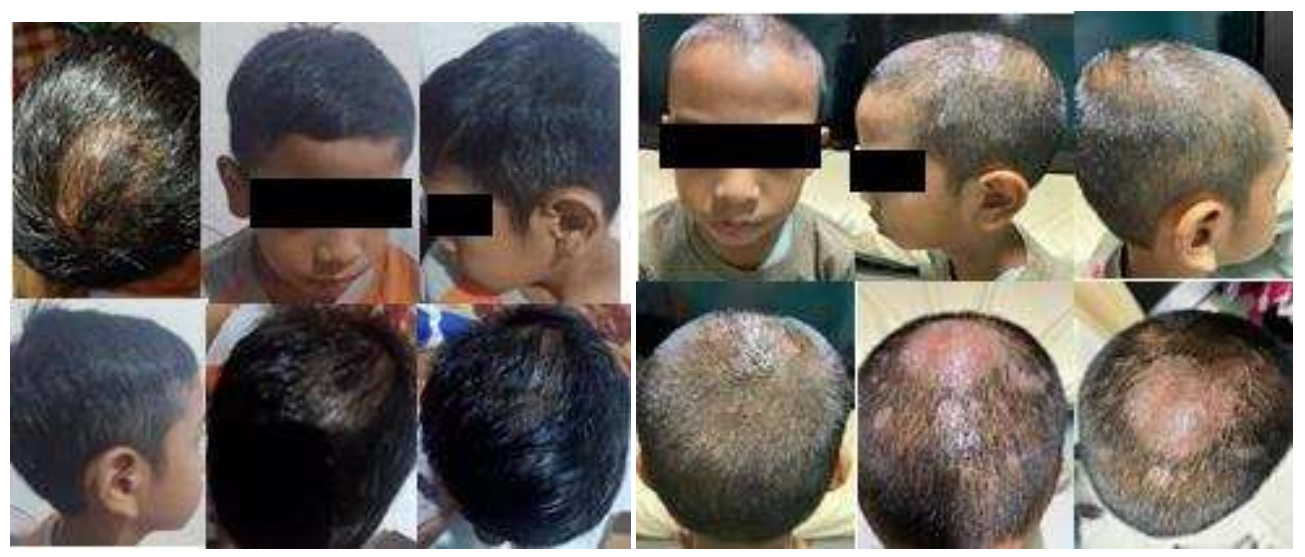

Figure 1. (a) Before treatment, (b) After treatment

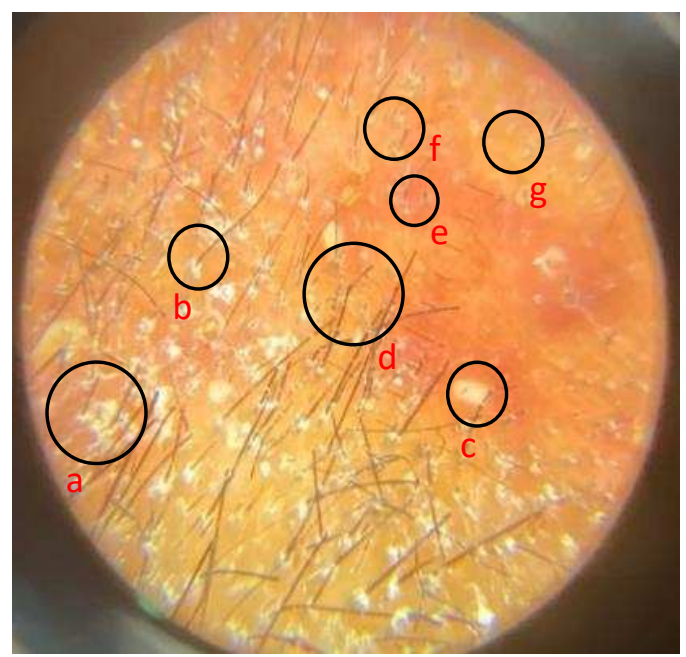

Figure 2. The trichoscopy result: a) Diffuse scaling, b) Perifollicular scaling, c) Morse code-like hair, d) Zigzag hair, e) Black dot, f) Broken hair, g) Comma hair 


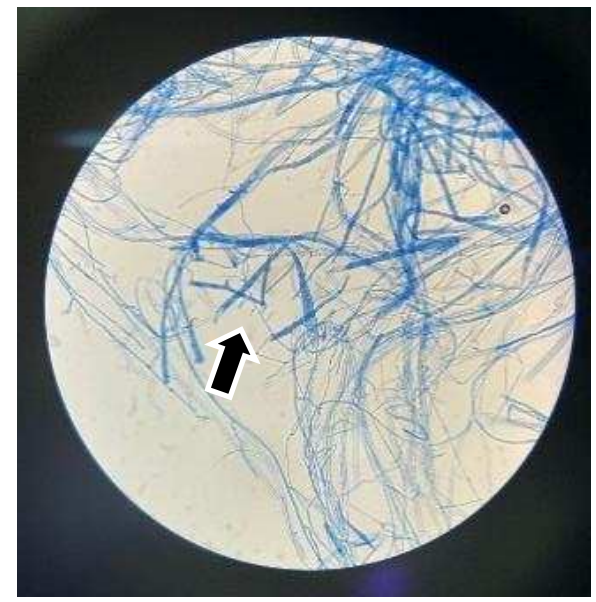

Figure 3. Lactofenol cotton blue staining showed macroconidia of M.canis with terminal knobs and more than 6 cells

\section{References}

1. Goldsmith LA, Katz SI, Gilchrest barbara A, Paller AS, Leffel DJ, Wolff K. Fitzpatrick's Dematology in General Medicine. 8th ed. New York: McGraw-Hill; 2011.

2. Andrews MD, Burns M. Common tinea infections in children. Am Fam Physician. 2008;77(10):1415-20.

3. Rodríguez-Cerdeira C, Martínez-Herrera E, Szepietowski JC, Pinto-Almazán R, Frías-De-León MG, Espinosa-Hernández VM, et al. A systematic review of worldwide data on tinea capitis: analysis of the last 20 years. J Eur Acad Dermatology Venereol. 2020;

4. Bhat Y, Zeerak S, Kanth F, Yaseen A, Hassan I, Hakak R. Clinicoepidemiological and mycological study of tinea capitis in the pediatric population of kashmir valley: A study from a tertiary care centre. Indian Dermatol Online J. 2017;8(2):100.

5. Alkeswani A, Cantrell W, Elewski B. Treatment of Tinea Capitis. Ski Appendage Disord. 2019;5(4):201-10.

6. Ely JW, Rosenfeld S, Stone MS. Diagnosis and Management of Tinea Infection. Am Fam Physician. 2014;90(10):702-10.

7. Ginter-Hanselmayer G, Smolle J, Gupta A. Itraconazole in the treatment of tinea capitis caused by Microsporum canis: Experience in a large cohort. Pediatr Dermatol. 2004;21(4):499-502.

8. Brasileiro A, Campos S, Cabete J, Galhardas C, Lencastre A, Serrão V. Trichoscopy as an additional tool for the differential diagnosis of tinea capitis: a prospective clinical study. Br J Dermatol. 2016;175(1):208-9. 
9. Xiao H, Pradhan S, Ran X, Ran Y. Tinea capitis: dermoscopy and calcium fluorescent microscopy as highly efficient and precise diagnostic tools. An Bras Dermatol. 2020;95(3):332-5.

10. James SL, Abate D, Abate KH, Abay SM, Abbafati C, Abbasi N, et al. Global, regional, and national incidence, prevalence, and years lived with disability for 354 Diseases and Injuries for 195 countries and territories, 1990-2017: A systematic analysis for the Global Burden of Disease Study 2017. Lancet. 2018;392(10159):1789-858.

11. Veasey JV, Muzy G de SC. Tinea capitis: Correlation of clinical presentations to agents identified in mycological culture. An Bras Dermatol. 2018;93(3):465-6.

12. Gürtler TGR, Diniz LM, Nicchio L. Tinea capitis micro-epidemic by Microsporum canis in a day care center of Vitória - Espírito Santo (Brazil). An Bras Dermatol. 2005;80(3):267-72.

13. Sari AB, Widaty S, Bramono K, Miranda E, Ganjardani M. TINEA KAPITIS DI POLIKLINIK KULIT DAN KELAMIN RSUPN DR CIPTO MANGUNKUSUMO JAKARTA PERIODE TAHUN 2005 - 2010. 2012;39(3):113-7.

14. Tan CW, Foong AY, Liew HM, Koh MJ. A Review of Tinea Capitis in a Cohort of Asian Children. Ann Acad Med Singapore. 2018;47(4):156-8.

15. Kakourou T, Uksal U, Oranje AP. Guidelines for the management of tinea capitis in children. Pediatr Dermatol. 2010;27(3):226-8. 